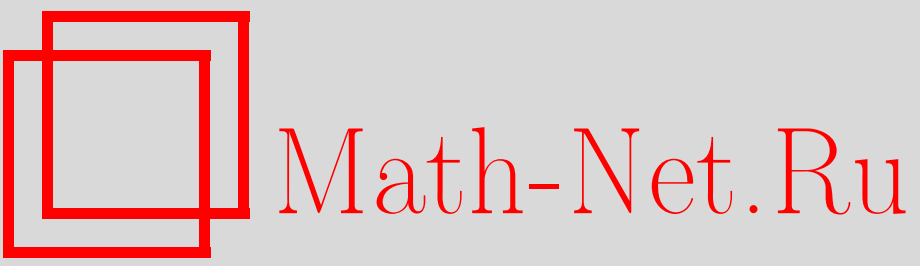

В. Р. Крым, Гладкие многообразия кинематического типа, ТМФ, 1999, том 119, номер 2, 264-281

DOI: https://doi.org/10.4213/tmf738

Использование Общероссийского математического портала Math-Net.Ru подразумевает, что вы прочитали и согласны с пользовательским соглашением

http://www . mathnet.ru/rus/agreement

Параметры загрузки:

IP : 54.198 .55 .26

26 апреля 2023 г., 09:27:30 
ТЕОРЕТИЧЕСКАЯ

И МАТЕМАТИЧЕСКАЯ

ФИЗИКА

Том 119, № 2

май, 1999

(C) 1999 г.

\section{ГЛАДКИЕ МНОГООБРАЗИЯ КИНЕМАТИЧЕСКОГО ТИПА}

Предлагается общий подход к описанию различных отношений типа причинности на гладких многообразиях. Каузальная структура может быть задана либо аксиоматически (конусом в касательном пространстве), либо псевдометрикой сигнатуры $(+-\ldots-)$ или $(+-\ldots-0 \ldots 0)$. В последнем случае многообразие приобретает структуру расслоенного пространства со слоями "абсолютной одновременности". Гладкая структура (атлас) многообразия непосредственно связана с его каузальной структурой.

\section{1. ВВЕДЕНИЕ}

1.1. Данная статья посвяшена рассмотрению отношений типа причинности на гладком многообразии. Интерес к этому направлению возник благодаря созданию специальной и общей теории относительности, однако далеко не все типы причинности (каузальной структуры) на многообразии были рассмотрены до настоящего времени. В данной статье мы восполним этот пробел. Кроме того, будет показано, что между каузальной и гладкой структурами на многообразии имеется определенная связь, существенная при построении теорий, имеющих целью объединить фундаментальные взаимодействия, включая гравитацию.

1.2. Напомним вкратце, с какими проблемами столкнулись предыдущие работы, направленные на объединение фундаментальных взаимодействий. Исторически первой работой на эту тему была статья Калущы [1], однако построенная теория носила сушественно приближенный характер. В 1926 г. Фок [2] показал, что траектория движения заряженной частицы может быть строго описана как геодезическая линия нулевой длины ("световая") в пятимерном многообразии. Клейн [3] независимо от Фока использовал тот же самый метрический тензор на пятимерном многообразии для вывода четырехмерных уравнений Эйнштейна и уравнений Максвелла. Клейн не рассматривал уравнений геодезических, а ограничился замечанием, что уравнения движения частиц обычным образом следуют из уравнений Эйнштейна. Во всех этих работах считалось, что

\footnotetext{
* Санкт-Петербургский государственный университет, Санкт-Петербург, Россия
} 
метрический тензор имеет сигнатуру $(+----)$, т.е. пятая координата пространственноподобна. Предполагалось, что метрический тензор не зависит от пятой координаты и что $g_{55}=1$. Оба эти условия неинвариантны.

В 1956 г. Румер [4] попытался отказаться от неинвариантного условия $g_{55}=1$. Пятую координату он также считал пространственноподобной. Румер получил классические уравнения движения заряженной частицы, но (как и Фок) только в предположении, что в пятимерном многообразии они являются геодезическими нулевой длины, т.е. световыми. Это обстоятельство совершенно непонятно с физической точки зрения. Румер выписал в явном виде уравнения Эйнштейна для пятимерного пространства с введенным им метрическим тензором. K сожалению, полученная Румером система уравнений неэквивалентна уравнениям Максвелла и Эйнштейна в присутствии электромагнитного поля.

Эйнштейн и Бергман в 1938 г. [5] предложили считать пространство-время циклически замкнутым в направлении пятой координаты. Они сделали это, для того чтобы обосновать независимость всех наблюдаемых физических величин от пятой координаты. Следуя Клейну и Фоку, период в направлении пятой координаты можно принять равным $\hbar / m c$. Румер также считает пространство-время замкнутым как цилиндр в направлении пятой координаты, но с периодом $\hbar$. Однако у Румера пятая координата имеет физический смысл и размерность действия, а объявлять действие циклическим некорректно, т.к. принцип наименшего действия при этом теряет смысл. Кроме того, действие не является независимой переменной. Еще более серьезное возражение против подхода Румера состоит в том, что пятимерное пространство-время он рассматривает как конфигурационное пространство для выбранной частицы, причем метрический тензор зависит от отношения заряда данной частицы к ее массе. Однако если бы каждая частица двигалась в своем собственном конфигурационном пространстве и общее для всех частиц пространство-время не имело бы физического смысла, то как могли бы частицы взаимодействовать между собой?

В 1960-е годы некоторым математикам, а именно Буземану [6] и Пименову [7] (см., кроме того, [8]), стало ясно, что дальнейшее обобщение теории относительности требует предварительного изучения отношения причинности на многообразиях. Для этого Буземан и Пименов предложили независимо довольно близкие наборы аксиом, из которых наиболее обшими следует признать аксиомы Пименова. Последний указывает, что дополнительная координата не может быть ни времени-, ни пространственноподобной: “Обычная риманова геометрия пригодна для моделирования величин только двух наименований: вешественного и мнимого. Физически это отвечает моделированию только двух физических размерностей. Но теория электромагнетизма, кроме размерности "время" и размерности "пространство", встречается еше по крайней мере с одной размерностью “электричество”. Поэтому, в широком смысле слова, теория электромагнетизма и невырожденная риманова геометрия не гомологичны" [7, с. 424].

На этой основе Пименов строит объединенную теорию гравитационных и электрома- 
гнитных взаимодействий. Пятая координата у Пименова считается независимой, как и должно быть в геометрии. Однако Пименову не удалось получить классических уравнений движения заряженной частишы. Кроме того, в его работе не используется принцип наименьшего действия. В уравнения типа Эйнштейна Пименов вводит формально построенный объект, который не является тензором Риччи ни для одной из двух рассматриваемых в его работе связностей. Поэтому, хотя в результате и получается система уравнений Эйнштейна и Максвелла, вывод этих уравнений у Пименова был проведен с нарушением традиший математической физики.

\section{2. ГЛАДКИЕ МНОГООБРАЗИЯ С ОТНОШЕНИЕМ ПРИЧИННОСТИ}

В линейном пространстве отношение частичного порядка следует выбирать так, чтобы оно было согласовано с линейной структурой и с топологией на этом пространстве $[9,10]$.

ОПРЕДЕЛЕНИЕ. Пусть $L$ - линейное пространство с топологией $\Delta$ и отношением частичного порядка $<$. Тройку $(L, \Delta,<)$ назовем линейной кинематикой, если она удовлетворяет следуюшим аксиомам:

1) отображение сложения $+: L \times L \rightarrow L$ непрерывно;

2 ) при любом $\alpha \in \mathbb{R}$ отображение умножения на $\alpha$ непрерывно;

3 ) отношение порядка транзитивно: из $a<b$ и $b<c$ следует, что $a<c$;

4) отношение порядка строгое: $a<a$ невозможно;

5) $\forall a, b \in L: a<b \Rightarrow \forall u \in L \quad a+u<b+u$;

6) $\forall a, b \in L: a<b \Rightarrow \forall \alpha \in \mathbb{R}_{+} \alpha a<\alpha b$, где $\mathbb{R}_{+}=(0,+\infty)$;

7) $\forall a \in L$ и $\forall U \in \Delta: a \in U \Rightarrow \exists b \in U: a<b$;

8) прошлое нулевой точки $Q_{0}^{+}:=\{u \in L \mid u<0\}$ открыто.

Обсудим вкратце, как устроено отношение частичного порядка, удовлетворяющее указанному набору аксиом. В линейном пространстве $L$ (возможно, бесконечномерном) множество точек, следуюших за данной точкой $a \in L$, образует выпуклый конус $Q_{a}^{+}$с вершиной $a$, причем $a \notin Q_{a}^{+}$. Множество точек $Q_{0}^{+}$, следуюших за нулевой точкой $0 \in L$, называется конусом будущего кинематики $L$. Для любой точки $a \in L$ имеем $Q_{a}^{+}=a+$ $Q_{0}^{+}$. Множество точек, предшествуюших данной точке $a \in L$, обозначается $Q_{a}^{-}$, причем $Q_{0}^{-}=-Q_{0}^{+}$и $\forall a \in L$ имеем $Q_{a}^{-}=a+Q_{0}^{-}$.

Топология $\Delta$ линейного пространства $L$ выбирается так, чтобы конус будушего $Q_{0}^{+}$ был открыт. Если пространство $L$ конечномерно, то это позволяет установить связь между типом конуса будущего $Q_{0}^{+}$и топологией в $L$. Именно, если конус будущего содержит прямые (такой конус напоминает "конек крыши”), то слабейшая топология в $L$ антидискретна на любой прямой, содержащейся в конусе будущего. Если же аффинная размерность конуса будушего меньше размерности $L$, то топология в $L$ содержит дискретные слои. Если конус будушего острый (т.е. не содержит прямых), имеет ту же 
размерность, что и $L$, и его гранища не содержит лучей, то слабейшая топология в $L$ евклидова.

Наша цель - перенести отношение частичного порядка с конечномерной линейной кинематики $(L,<, \Delta)$ на гладкое многообразие $M$. При этом кинематика $L$ будет использоваться в качестве картируюшего множества ("модели”). Для того чтобы понятия непрерывности и гладкости отображений на многообразиях не зависели от типа конуса будушего в $L$, в следуюшем определении мы выбираем в $L$ евклидову топологию. В евклидовой топологии конус будущего кинематики $L$ открыт в своей аффинной оболочке, если только граница этого конуса не содержит лучей.

2.1. ОПРЕДЕЛЕНИЕ. Пусть $M$-гладкое многообразие, $L$ - линейная кинематика той же размерности. Пусть на $M$ задано семейство непрерывных отображений $(P(U))_{U \in I}$, $P(U): T U \rightarrow L \forall U \in I$, причем каждый элемент $U \in I$ - связное открытое подмножество в $M$, таких, что:

1) $\bigcup_{U \in I} U=M$ (т.е. $I$ - покрытие многообразия $\left.M\right)$;

2) $\forall U \in I$ и $\forall x \in U$ сужение $P(U)_{x}: T_{x} U \rightarrow L$ - невырожденное линейное отображение;

3) $\forall U, V \in I$ и $\forall x \in U \cap V$ отображение перехода $P(U)_{x} \circ P(V)_{x}^{-1}: L \rightarrow L$ сохраняет порядок в $L$.

Тогда $M$ с указанным семейством отображений $P$ назовем многообразием кинематического типа $L$. Семейство $P$ будем назьвать кинематическим атласом на $M$.

В каждом касательном пространстве $T_{a} M$ многообразия кинематического типа $L$ корректно определен конус $P(U)_{a}^{-1}\left(Q_{0}^{+}\right)$, являющийся прообразом конуса будущего нулевой точки кинематики $L$ при отображении $P(U)_{a}$. В силу условия 3 этот конус не зависит от выбора области $U(a \in U)$, поэтому обозначение области $U$ мы будем опускать. Обозначим $Q_{a}^{+}:=P_{a}^{-1}\left(Q_{0}^{+}\right)$и $Q_{a}^{-}:=P_{a}^{-1}\left(Q_{0}^{-}\right)$. Семейство конусов $Q_{a}^{+}$(и $\left.Q_{a}^{-}\right)$ непрерывно зависит от точки $a \in M$ в том смысле, что отображение $P(U)$ непрерывно.

ПримеР 1. Любая линейная кинематика $L$ является многообразием кинематического типа $L$, кинематический атлас которого состоит только из одного отображения $P_{x}:=i d_{L}-x \forall x \in L$. Отношение порядка (т.е. конус будушего $Q_{0}^{+}$) кинематики $L$ выбирается произвольно, но так, чтобы оно удовлетворяло некоторым естественным аксиомам $[9,10]$. Ньютоново отношение причинности задается условием $t>0$, т.е. конусом будушего является полупространство, а эйнштейново отношение причинности задается эллиптическим конусом. Эти отношения причинности определяют разные слабейшие топологии кинематики $L$ : для ньютоновой причинности это естественная топология $\mathbb{R}^{1} \times \mathbb{R}_{a}^{n-1}$, где $\mathbb{R}_{a}$ - вешественная прямая с антидискретной топологией, $n:=\operatorname{dim} L$, a для эйнштейновой причинности слабейшая топология евклидова. Эти типы причинности не исчерпывают всех возможностей.

ПримеР 2. Пусть $M$ - параллелизуемое многообразие размерности $n$ и $X_{1}, \ldots, X_{n}-$ линейно независимые в каждой точке непрерывные векторные поля на $M$. Пусть $L-$ 
линейная кинематика той же размерности. Отображение $P: T M \rightarrow L, P(Y):=\left(Y^{1}, \ldots\right.$ $\left.\ldots, Y^{n}\right)$, где

$$
Y=\sum_{k=1}^{n} Y^{k} X_{k}
$$

определено на всем $M$. Пара $(M, P)$ является многообразием кинематического типа $L$.

\section{2. Ориентация времени.}

ОПредЕЛЕниЕ. Пусть $(M, P)$ - многообразие кинематического типа $L$. Ориентацией времени многообразия $M$ называется непрерывное векторное поле $X$ на $M$ такое, что $\forall a \in M \quad X(a) \in Q_{a}^{+}$. В частности, поле $X$ нигде не обрашается в нуль.

Tеорема 1. На любом многообразии $(M, P)$ кинематического типа L существует ориентация времени.

ДокАЗАТЕльСтво. Выберем вектор $\nu \in Q_{0}^{+} \subset L$. У любой точки $a \in M$ сушествует окрестность $U$, на которой определено отображение $P(U)$ семейства $P$. Поэтому на $U$ имеется непрерывное векторное поле $Y_{U}, \forall x \in U \quad Y_{U}(x):=P(U)_{x}^{-1}(\nu)$. Пусть $\left(\chi_{U}\right)_{U \in I}$ - разложение единицы, ассоциированное с покрытием $I$. Поскольку многообразие $M$ локально компактно, можно считать, что покрытие $I$ локально конечно. В силу выпуклости конуса $Q_{a}^{+}$векторное поле

$$
X:=\sum_{U \in I} \chi_{U} Y_{U}
$$

- то, которое требуется (в этой сумме в каждой точке только конечное число слагаемых отлично от нуля).

2.3. Обсудим достаточные для дальнейшего примеры отображений перехода $P(U)_{x}$ ○ $P(V)_{x}^{-1}$ для различных типов линейных кинематик $L$.

2.3.1. Пусть конус будушего $Q_{0}^{+} \subset L$ эллиптический. Тогда в силу известных теорем всякое линейное отображение, сохраняющее конус $Q_{0}^{+}$, является преобразованием Лоренца с произвольной мультипликативной постоянной, т.е. линейные отображения, сохраняющие $Q_{0}^{+}$, ортогональны в псевдометрике сигнатуры $(+-\cdots-)$, которая однозначно восстанавливается по конусу $Q_{0}^{+}$с точностью до масштабируюшего множителя.

2.3.2. Пусть конус будушего $Q_{0}^{+}$содержит хотя бы одну прямую. Тогда $[9,10]$ слабейшая топология кинематики $L$ содержит нетривиальное линейное антидискретное подпространство. Рассмотрим максимальное линейное подпространство $l_{0}$, содержашееся в замыкании конуса будушего $Q_{0}^{+}$кинематики $L$. Пусть $n:=\operatorname{dim} L, k:=\operatorname{dim} l_{0}$. Пусть $e_{n-k+1}, \ldots, e_{n}$ - базис подпространства $l_{0}$. Подпространство $l_{0}$ переходит в себя при линейных преобразованиях, сохраняюших порядок. Поэтому в системе координат, в которой координата с номером $j$ определена как коэффициент при $e_{j} \quad(j=n-$ 
$k+1, \ldots, n)$, матрица отображения перехода $P(U)_{x} \circ P(V)_{x}^{-1}$ имеет блочный вид

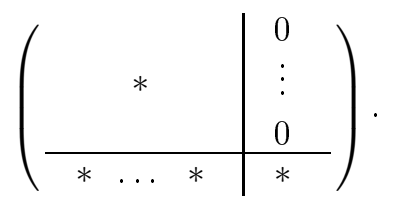

Левый верхний блок (квадратный, размерности $n-k$ ) является невырожденным линейным преобразованием. Правый верхний блок нулевой. Правый нижний блок (квадратный, размерности $k$ ) также является невырожденным линейным преобразованием. Известно, что такие матришы образуют группу.

2.3.3. Пусть размерность конуса будушего $Q_{0}^{+}$кинематики $L$ меньше размерности пространства $L$. Тогда $[9,10]$ слабейшая топология кинематики $L$ содержит нетривиальное линейное дискретное подпространство. Минимальное линейное подпространство $l_{0}$, содержашее конус будушего $Q_{0}^{+}$, переходит в себя при линейных преобразованиях, сохраняюших порядок. Пусть $n:=\operatorname{dim} L, k:=\operatorname{dim} l_{0}, e_{1}, \ldots, e_{k}-$ базис подпространства $l_{0}$. Тогда матрица отображения перехода $P(U)_{x} \circ P(V)_{x}^{-1}$ имеет блочный вид

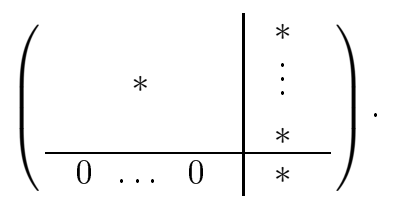

Левый верхний блок (квадратный, размерности $k$ ) является невырожденным линейным преобразованием. Левый нижний блок нулевой. Правый нижний блок (квадратный, размерности $n-k$ ) также является невырожденным линейным преобразованием. Такие матрицы образуют группу.

2.3.4. Множество линейных изоморфизмов $L \rightarrow L$, сохраняюших частичный порядок в $L$, является группой, которую мы обозначим $G L(L,<)$. На многообразии $M$ могут существовать такие кинематические атласы $(P(U))_{U \in I}$, что все отображения перехода принадлежат некоторой подгруппе $H$ этой группы. Например, на параллелизуемом многообразии (пример 2) сушествует кинематический атлас, состоящий из одного отображения, и подгруппа $H$ единичная. Верно и обратное: если на многообразии существует кинематический атлас такой, что все отображения перехода тождественные, то многообразие параллелизуемо.

Множество линейных изоморфизмов $L \rightarrow L$, сохраняюших определенные в пунктах 2.3.2 и 2.3.3 линейные подпространства $l_{0}$, обозначим $G L(L, \operatorname{Lin}(<))$. Это подгруппа группы $G L(L,<)$.

\section{4. Локальный порядок.}


2.4.1. ОПРЕДЕЛЕНИЕ. Пусть $(M, P)$ - многообразие кинематического типа $L$, $\gamma:[a, b] \rightarrow M-$ кусочно-гладкий путь. Путь $\gamma$ называется направленным в будущее, если $\forall t \in[a, b] \gamma^{\prime}(t) \in Q_{\gamma(t)}^{+}$. Если путь $\gamma$ не дифференцируем в точке $t$, то этому условию должны удовлетворять обе односторонние производные в точке $t$. Аналогично определяется путь, направленный в прошлое. Путь $\gamma$ называется времениподобным, если он направлен в будушее или в прошлое.

ОПрЕДЕЛЕНИЕ. Пусть $(M, P)$ - многообразие кинематического типа $L, U \subset M$, $x, y \in U$ и существует направленный в будущее путь $\gamma:[a, b] \rightarrow U$ с началом $x$ и концом $y$ (т.е. $\gamma(a)=x, \gamma(b)=y)$. Тогда обозначим $x<y(\bmod \gamma)$. Аналогично, если путь $\gamma$ направлен в прошлое, то обозначим $x>y(\bmod \gamma)$.

Очевидно, что $x<y(\bmod \gamma) \Leftrightarrow y>x\left(\bmod \gamma^{-1}\right)$ (достаточно пройти путь $\gamma$ в обратную сторону).

Отношение < хотелось бы интерпретировать как локальный порядок на $M$, однако такая интерпретация не всегда возможна.

2.4.2. Пусть $U \subset M$ - некоторая область, и пусть $(M, P)$ - многообразие кинематического типа $L$ такое, что замыкание конуса будущего кинематики $L$ содержит нетривиальное линейное подпространство $l_{0}$. Тогда на $M$ возникает распределение $l_{x}:=P_{x}^{-1}\left(l_{0}\right) \forall x \in M$. Предположим, что распределение $\left(l_{x}\right)_{x \in U}$ гладкое и вполне неголономное. Тогда на $U$ сушествуют гладкие векторные поля $X$ и $Y$ такие, что $X(a)$, $Y(a) \in l_{a} \forall a \in U$, но $[X, Y]_{a} \notin \mathrm{Cl} Q_{a}^{+} \forall a \in U$. Известно, что движение по интегральной кривой векторного поля $[X, Y]$ можно реализовать с помощью последовательных смешений вдоль интегральных кривых векторных полей $X$ и $Y$. Поскольку $X(a), Y(a) \in$ $\mathrm{Cl} Q_{a}^{+} \forall a \in U$, смешения вдоль этих интегральных кривых можно сколь угодно точно аппроксимировать движением вдоль времениподобных путей в $U$, направленных в будушее. Однако тогда $\forall a, b \in U \quad \exists \gamma: a<b(\bmod \gamma)$ как бы ни мала была область $U$. В частности, в $U$ имеются замкнутые времениподобные пути (т.е. такие, у которых начало и конец совпадают).

Теорема 2. Пусть $(M, P)$ - многообразие кинематического типа L. Обозначим через $l_{0}$ максимальное линейное подпространство, содержащееся в замыкании конуса будущего кинематики L. Пусть распределение $l_{x}:=P_{x}^{-1}\left(l_{0}\right), \quad x \in M$, голономно. Тогда для любой точки $x \in M$ существует окрестность $U$ такая, что любой времениподобньй путь в $U$ не имеет точек самопересечения.

Если выполнены условия теоремы 2 и область $U$ такая, как указано в теореме, то мы будем использовать обозначение $a<b(\bmod U)$. Отношение $<$ в этих условиях перестает зависеть от выбора пути $\gamma$, а зависит только от выбора области $U$.

ДоКАЗАТЕЛЬСТВо. На достаточно малой области $U$ голономное распределение $l$ можно расширить до распределения $S$ такого, что:

1) $\forall x \in U l_{x} \subset S_{x}$; 
2) $\forall x \in U S_{x}$ отделяет $Q_{x}^{+}$от $Q_{x}^{-}$;

$3)$ распределение $S$ имеет коразмерность 1 и голономно.

Выберем произвольную точку $x \in M$. Конусы $Q_{x}^{+}$и $Q_{x}^{-}$выпуклы, не пересекаются, и, следовательно, их можно отделить гиперплоскостью $s_{x}$ (очевидно, что $l_{x} \subset s_{x}$ ). Эту гиперплоскость можно выбрать так, чтобы $s_{x} \cap\left(\mathrm{Cl} Q_{x}^{+} \cup \mathrm{Cl} Q_{x}^{-}\right)=l_{x}$. Гиперплоскость $s_{x}$ является касательным пространством в точке $x$ к некоторому гладкому подмногообразию $N \subset M$ коразмерности 1 . Подмногообразие $N$ может быть выбрано так, чтобы оно содержало все интегральные подмногообразия распределения $l$, проходяшие через любую точку $N$. Например, пусть $n:=\operatorname{dim} M, k:=\operatorname{dim} l_{0}$. В некоторой окрестности $W$ точки $x$ сушествует $(n-k-1)$-мерное гладкое подмногообразие $P$, трансверсальное к распределению $l$ в $W$. Множество всех интегральных подмногообразий распределения $l$, проходяших через $P$, является требуемым многообразием $N$ коразмерности 1 (для достаточно малой области $W$ ).

Рассмотрим "распределение" $S$ касательных плоскостей к подмногообразию $N$. Поскольку семейства конусов $\left(Q_{x}^{+}\right)_{x \in U}$ и $\left(Q_{x}^{-}\right)_{x \in U}$ непрерывно зависят от точки $x$, то на достаточно малой области $W$ гиперплоскости $s_{x}$ отделяют $Q_{x}^{+}$и $Q_{x}^{-}$для всех $x \in N$.

Рассмотрим семейство интегральных подмногообразий распределения $l$ на $W$. Имеется диффеоморфизм $\varphi: W \rightarrow \mathbb{R}^{n}$ такой, что отображение $\varphi$ переводит семейство интегральных подмногообразий распределения $l$ на $W$ в семейство линейных подпространств в $\mathbb{R}^{n}$. Действительно, указанное семейство подмногообразий является $(n-k)$-параметрическим $C^{1}$-гладким семейством. Локально оно может быть задано набором отображений $f_{r}: \mathbb{R}^{k} \rightarrow M, r \in \mathbb{R}^{n-k}$, причем отображение $f$ является $C^{1}$-диффеоморфизмом. Заменим карту на множестве $W$ : координаты точки $a \in W$ суть $(r, s)$ тогда и только тогда, когда $a \in \operatorname{im} f_{r}$ и $f_{r}(s)=a$. В этой карте семейство интегральных подмногообразий распределения $l$ задается уравнением $r(a)=r_{0}$, т.е. является семейством $k$-мерных плоскостей в $\mathbb{R}^{n}$. Все эти подпространства - эквидистантные поверхности в евклидовой метрике $\operatorname{Diag}(1, \ldots, 1)$. Дифференциал гладкого отображения $\varphi: W \rightarrow \mathbb{R}^{n}$, определенного равенством $\varphi(a):=(r, s)$, переносит эту метрику в $W$ :

$$
\langle u, \nu\rangle:=\left(d_{x} \varphi(u), d_{x} \varphi(\nu)\right) \quad \forall u, \nu \in T_{x} W .
$$

В указанной римановой метрике $\langle$,$\rangle на W$ интегральные подмногообразия распределения $l$ являются эквидистантными поверхностями.

Построим семейство эквидистантных поверхностей (в указанной римановой метрике) для подмногообразия $N \subset W$ коразмерности 1 , определенного вьше. Обозначим семейство этих поверхностей $\left(N_{\varepsilon}\right)_{\varepsilon \in(-\delta, \delta)}, \delta>0$. Они обладают следуюшим свойством: каждое подмногообразие $N_{\varepsilon}$ содержит все интегральные подмногообразия распределения $l$, проходящие через любую точку из $N_{\varepsilon}$.

Поскольку семейства конусов $\left(Q_{x}^{+}\right)_{x \in W}$ и $\left(Q_{x}^{-}\right)_{x \in W}$ непрерывно зависят от точки $x$, имеется окрестность $U$ точки $x$ такая, что касательные пространства $T_{x} N_{\varepsilon}$ отделяют 
конусы $Q_{x}^{+}$и $Q_{x}^{-}$для всех $x \in U$. Обозначим распределение касательных пространств $T_{x} N_{\varepsilon}(x \in U)$ символом $S$.

Область $U$ может быть выбрана настолько малой, что для любой точки $x \in U$ интегральное подмногообразие распределения $S$, проходяшее через $x$, делит $U$ на две компоненты связности.

Пусть $\gamma-$ путь в $U$, направленный в будущее. Рассмотрим максимальное на $U$ интегральное подмногообразие распределения $S$, проходящее через точку $x \in \operatorname{im} \gamma$. Это подмногообразие делит $U$ на две компоненты связности. Путь $\gamma$ начинается в одной из этих компонент и заканчивается в другой. Следовательно, замкнутых времениподобных путей в $U$ нет.

В дальнейшем мы будем рассматривать только такие многообразия кинематического типа $(M, P, L)$, которые удовлетворяют условиям теоремы 2.

2.4.3. СвоЙствА ОТношЕНИЯ $<$ :

1) отношение < топологически не пусто, т.е. в любой окрестности $U$ любой точки $a \in M$ найдутся точки $x, y \in U$ такие, что $x<y(\bmod U)$ (достаточно проинтегрировать введенную в пункте 2.2 ориентацию времени);

2) отношение < антирефлексивно, т.е. для любой точки $x \in M$ существует окрестность $U \ni x$ такая, что $-(x<x(\bmod U))$;

3) отношение < локально транзитивно, т.е. для любой точки а $\in M$ существует окрестность $U$ такая, что $\forall x, y, z \in U: x<y(\bmod U) u y<z(\bmod U) \Rightarrow$ $x<z(\bmod U)$.

ОПРЕДЕЛЕНИЕ. Пусть $M-$ многообразие, $<-$ отношение на $M$ со свойствами $1-3$. Тогда < называется локальным порядком на $M$.

Итак, на всяком многообразии $(M, P)$ кинематического типа $L$ определен локальный порядок, зависяший от частичного порядка в $L$ и кинематического атласа $P$. Мы не будем рассматривать глобальное транзитивное замыкание отношения $<$, т.к. такое замыкание может не быть отношением порядка (даже локальным) [11]. Например, на окружности можно задать локальный порядок, но не глобальный.

2.4.4. Локальный порядок < задает на многообразии отношение локальной эквивалентности, которая вводится следующим образом.

ОПРЕДЕЛЕНИЕ. Пусть $x, y \in M, U \ni x$ - некоторая область на многообразии $M$, удовлетворяющая условиям теоремы 2. Обозначим $K_{x}^{+}:=\{a \in U \mid a>x(\bmod U)\}$, $K_{x}^{-}:=\{a \in U \mid a<x(\bmod U)\}$. Примем, что $x \leqslant y(\bmod U)$ тогда и только тогда, когда $x \in \mathrm{Cl} K_{y}^{-}$, и $x \approx y(\bmod U)$ тогда и только тогда, когда $x \leqslant y$ и $y \leqslant x$.

СвоЙСТВА ОТНОшЕНИЯ $\approx$ :

1) рефлексивность, которая следует из того, что в любой окрестности данной точки есть точки прошлого и будущего данной точки в смисле локального порядка <; отношение $\leqslant$ также рефлексивно; 
2) симметричность, следующая непосредственно из определения;

3) транзитивность, следующая из транзитивности отношения $\leqslant$.

В классической специальной теории относительности, где конус будущего эллиптический, отношение $\approx$ тривиально: класс $x / \approx$ эквивалентности любой точки $x$ состоит в точности из этой точки. В ньютоновой кинематике, где конусом будущего является полупространство, отношение $\approx$ трактуется как отношение одновременности. Класс $x / \approx$ любой точки $x$ - гиперплоскость.

2.4.5. Известно, что всякий конус, содержащий прямые, является декартовым произведением острого конуса и аффинного подпространства. Поэтому, если конус будушего кинематики $L$ содержит прямую, то на многообразии $(M, P)$ кинематического типа $L$ имеется распределение размерности $m$, где $m$ - размерность максимального линейного подпространства, содержашегося в замыкании конуса будушего (в естественной топологии кинематики $L$ это подпространство антидискретное). Пусть область $U$ удовлетворяет условиям теоремы 2 . Тогда для любой точки $x \in U$ класс $x / \approx$ является подмногообразием, замкнутым в $U$. Это в точности максимальное (в $U)$ интегральное многообразие указанного распределения, проходящее через точку $x$. Действительно, для любого пути, вектор скорости которого принадлежит распределению, найдутся сколь угодно близкие к нему пути, направленные в прошлое и будушее. Топологическое пространство $U / \approx$ является многообразием.

Обозначим через $\sim$ транзитивное замыкание отношения $\approx$ на всем $M$. Класс $x / \sim$ может не быть замкнутым в $M$, и фактор-пространство $M / \sim$ может не быть многообразием, даже если указанное распределение одномерно.

ПримеР 3 . Пусть $M$ - цилиндр $S^{1} \times \mathbb{R}$. Зададим на $M$ отображение $P$ векторов $(u, \nu) \in T_{(\alpha, x)} M:$

$$
P_{(\alpha, x)}(u, \nu):= \begin{cases}\left(\begin{array}{l}
u \\
\nu
\end{array}\right) & \left(x \geqslant x_{0}\right), \\
\left(\begin{array}{cc}
1 & x-x_{0} \\
x_{0}-x & 1
\end{array}\right) & \left(x \leqslant x_{0}\right),\end{cases}
$$

где $x$ - высота вдоль образуюшей цилиндра, $\alpha$ - угловая координата на окружности.

Выберем двумерную линейную кинематику $L$ с конусом $Q_{0}^{+}$в виде открытой полуплоскости (слабейшая топология в $L$ есть $\mathbb{R} \times \mathbb{R}_{a}$, где $\mathbb{R}_{a}$ - вешественная прямая с антидискретной топологией). Пара $(M, P)$ является многообразием кинематического типа $L$. Чтобы найти классы $x / \approx$, найдем прообраз антидискретного слоя кинематики $L$ при отображении $P$ :

$$
\left(\begin{array}{cc}
1 & x-x_{0} \\
x_{0}-x & 1
\end{array}\right)\left(\begin{array}{l}
1 \\
0
\end{array}\right)=\left(\begin{array}{c}
1 \\
x_{0}-x
\end{array}\right) .
$$

Это касательное векторное поле на $M$. Проинтегрируем его. Система дифференциальных уравнений $\dot{\alpha}=1, \dot{x}=x_{0}-x$ имеет обшее решение $\alpha=t+c_{1}, x=x_{0}-e^{-t+c}$. 


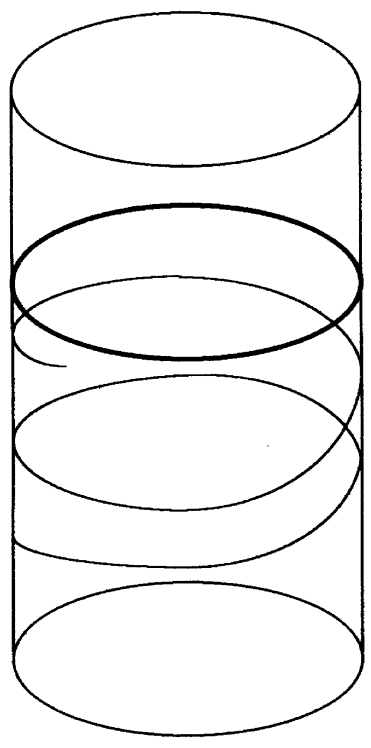

Рис. 1

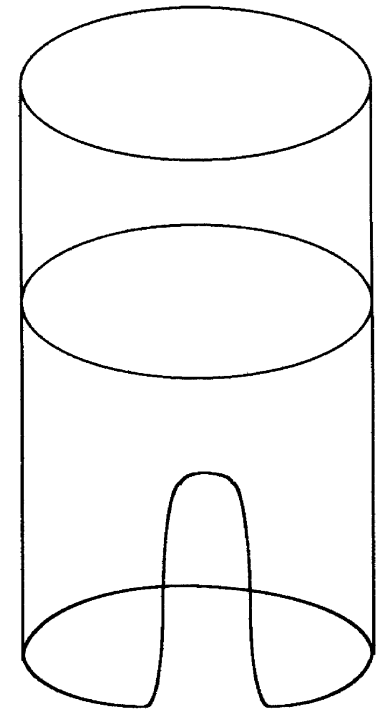

Рис. 2

Следовательно, классы отношения $\sim$ до высоты $x_{0}-$ спирали с бесконечным числом оборотов вокруг цилиндра, а выше $x_{0}$ - окружности. Фактор-пространство $M / \sim$ состоит из окружности и луча, содержашего свою вершину, причем любая окрестность вершины луча целиком содержит эту окружность. Это топологическое пространство не является многообразием и не вкладывается в $M$ (рис. 1).

ПРИмеР 4. Похожим образом можно превратить в многообразие кинематического типа $\mathbb{R} \times \mathbb{R}_{a}$ и “цилиндр с разрезом" (рис. 2). Пусть отображение $P$ такое же, как в примере 3 при $x \geqslant x_{0}$. Тогда фактор-пространство - просто $\mathbb{R}$ с обычным упорядочиванием, но классы эквивалентности $\sim$ различных точек, вообще говоря, не гомеоморфны друг другу.

2.5. Гладкие отображения многообразий кинематического типа. Пусть $N$ - гладкое многообразие, $(M, P)$ - многообразие кинематического типа $L$ и $\varphi: N \rightarrow M$ - гладкое отображение. Тогда на $N$ возникает кинематический атлас $(P(U) \circ d \varphi)_{U \in I}$. Он удовлетворяет условиям определения пункта 2.1 , только если в каждой точке $x \in N$ дифференциал $d_{x} \varphi$ обратим. Тогда $(N, P \circ d \varphi)$ также оказывается многообразием кинематического типа $L$.

ОПРЕДЕЛЕНИЕ. Пусть $(M, P)$ и $(N, T)$ - гладкие многообразия кинематического типа $L, P=(P(U))_{U \in I}, T=(T(V))_{V \in I^{\prime}}$. Говорим, что гладкое отображение $\varphi: N \rightarrow M$ есть диффеоморфизм многообразий кинематического типа, если $\varphi$ - диффеоморфизм $N$ 
и $M$ как гладких многообразий и

$\forall U \in I, \forall V \in I^{\prime} \quad$ и $\forall x \in V \quad \varphi(x) \in U \Rightarrow\left(P(U)_{\varphi(x)} \circ d_{x} \varphi\right)^{-1}\left(Q_{0}^{+}\right)=\left(T(V)_{x}\right)^{-1}\left(Q_{0}^{+}\right)$.

Таким образом, отображения $P(U)_{\varphi(x)} \circ d_{x} \varphi \circ\left(T(V)_{x}\right)^{-1}$ суть линейные автоморфизмы кинематики $L$, сохраняюшие частичный порядок в $L$. Отношения локального порядка на диффеоморфных многообразиях кинематического типа, очевидно, совпадают после отождествления этих многообразий с помошью диффеоморфизма $\varphi$. Будем говорить также, что $N$ и $M$ диффеоморфны с сохранением порядка.

2.6. Упорядочивание кокасательного пространства. Мы имеем отношение локального порядка на многообразии $M$ и частичный порядок на любом касательном пространстве $T_{x} M$. Нам понадобится также отношение порядка на кокасательном пространстве. Положим $\forall \omega \in T_{x}^{*} M \omega>0 \Leftrightarrow \forall u \in Q_{x}^{+} \subset T_{x} M \omega \cdot u>0$ (где действие ковектора на вектор обозначается точкой). Иными словами, порядок в кокасательном пространстве определяется конусом, дуальным к конусу будушего в касательном пространстве. Непосредственно проверяется, что существует топология на $T_{x}^{*} M$, для которой это пространство является линейной кинематикой. Вообще говоря, при таком переходе из касательного пространства в сопряженное кинематический тип меняется, причем антидискретный слой переходит в дискретное расслоение и наоборот. Только если слабейшая топология в кинематике $L$ евклидова, слабейшая топология в $L^{*}$ та же самая.

\section{3. ПСЕВДОРИМАНОВЫ МНОГООБРАЗИЯ}

3.1. На каждом псевдоримановом многообразии $M$ со скалярным произведением $\langle$, сигнатуры $(+-\ldots-)$ и ориентацией времени $X$ (непрерывным времениподобным векторным полем, не обращающимся в нуль) сушествует некоторая каноническая причинность. Она задается кинематическим атласом, определенным следуюшим образом.

У каждой точки $a \in M$ есть окрестность $U$, на которой геодезические с началом $a$ не пересекаются. Обозначим через $P_{x}(\nu)$ вектор, полученньй параллельным переносом вектора $\nu \in T_{x} M$ вдоль геодезической, соединяющей точки $x$ и $a$ в $U$, в точку $a$. Очевидно, что отображение $P$ непрерьвно и невырождено.

Обозначим $Q_{x}^{+}:=\left\{\nu \in T_{x} M \mid\langle\nu, \nu\rangle>0\right.$ и $\left.\langle X(x), \nu\rangle>0\right\}$.

ЛЕмма. Пусть связность $\nabla$ на $M$ риманова (т.е. $\quad X\langle Y, Z\rangle=\left\langle\nabla_{X} Y, Z\right\rangle+$ $\left\langle Y, \nabla_{X} Z\right\rangle$ для любых векторных полей $\left.X, Y, Z\right)$. Тогда параллельный перенос $P$ вдоль любой кривой $\gamma$ сохраняет порядок: $P_{x}\left(Q_{x}^{+}\right)=Q_{a}^{+}$.

ДокАЗАТЕЛЬСТво. Пусть $Y$-времениподобноепараллельное векторное поле вдоль пути $\gamma$. Тогда

$$
\frac{d}{d t}\langle Y, Y\rangle=2\left\langle\frac{D Y}{d t}, Y\right\rangle=0
$$


т.е. $\langle Y, Y\rangle=$ const вдоль $\gamma$. Поскольку два времениподобных вектора не могут быть взаимно ортогональны, функция $\langle Y, X\rangle$ не обращается в нуль вдоль $\gamma$ и, следовательно, сохраняет знак. Вместе с невырожденностью отображения $P$ это доказывает утверждение леммы.

Касательное пространство $T_{a} M$ в любой точке $a \in M$ можно выбрать в качестве линейной кинематики $L$, поскольку все они имеют одно и то же отношение порядка. Очевидно, что конус $Q_{a}^{+} \subset L$ эллиптический. Итак, у каждой точки $a \in M$ имеется окрестность $U$, на которой определено отображение $P$ такое, что пара $(U, P)$ является многообразием кинематического типа $L$. Если две такие области пересекаются, то соответствуюшие отображения согласованы между собой: $\forall x \in U \cap V P(U)_{x}^{-1}\left(Q_{0}^{+}\right)=$ $P(V)_{x}^{-1}\left(Q_{0}^{+}\right)$. Нами доказана следующая теорема.

ТЕОРема 3. Пусть $M-$ псевдориманово многообразие со скалярным произведением $\langle$,$\rangle сигнатуры (+-\cdots-)$ и ориентацией времени $X$ (непрерывным времениподобным векторным полем на $M$, не обращающимся в нуль). Пусть $P-$ семейство всех построенных выше отображений. Тогда $(M, P)$ является многообразием кинематического типа $L$, где в качестве $L$ можно взять любое касательное пространство $T_{a} M$ с суженной на него псевдометрикой, причем конус будущего этой кинематики въбирается так, чтобъ он содержсал вектор X $(a)$.

ЗАмечание. Риманову связность, указанную в лемме, можно выбирать по-разному. Можно также менять семейство кривых, вдоль которых осуществляется параллельный перенос. При этом будут получаться различные кинематические атласы $P$. Однако все многообразия $(M, P)$ будут диффеоморфны между собой как многообразия кинематического типа.

3.2. ПроБЛЕмА. При каких условиях по данному многообразию $(M, P)$ кинематического типа $L$ можсно построчть кинематический атлас $Z$ такой, что все отображения $Z(U): T U \rightarrow L$ являются дифференциалами некоторых гладких отображений $f: U \rightarrow L u(M, P)$ диффеоморфно $(M, Z)$ с сохранением порядка? (См. также [7, с. 351 и 416].)

Ясно, что положительное решение этой проблемы возможно далеко не на всяком многообразии. С другой стороны, существование кинематического атласа не накладывает слишком сильных ограничений на строение многообразия в целом.

ПримеР 5. Кинематическое упорядочивание можно ввести и на неориентируемом многообразии, например на листе Мебиуса. Достаточно отобразить конус будущего двумерной кинематики $L$ на $T M$ так, чтобы ось симметрии конуса была параллельна продольной линии листа Мебиуса. Слабейшая топология в $L$ может быть как $\mathbb{R} \times \mathbb{R}_{a}$, так и $\mathbb{R}^{2}$. Смена пространственной ориентации при движении конуса вдоль средней линии не имеет значения, т.к. ориентация времени сохраняется. 


\section{4. ПСЕВДОМЕТРИЧЕСКИЕ ГЛАДКИЕ МНОГООБРАЗИЯ}

Здесь мы рассмотрим псевдоримановы многообразия с несколько ослабленными требованиями к псевдометрике.

ОПрЕДЕЛЕНИЕ. Пусть $M$ - гладкое многообразие. Назовем $M$ псевдометрическим, если на $T M$ задана симметричная билинейная форма $\langle,\rangle_{x}: T_{x} M \times T_{x} M \rightarrow \mathbb{R}$ сигнатуры $(+-\ldots-0 \ldots 0)$, гладко зависяшая от точки $x \in M$. Форму $\langle$,$\rangle будем называть$ скалярным произведением.

Будут рассматриваться только такие псевдометрические многообразия $M$, на которых сушествует ориентация времени, т.е. непрерывное векторное поле $X$ такое, что всюду $\langle X, X\rangle>0$. Обозначим $F_{x}:=\left\{u \in T_{x} M \mid\langle u, u\rangle>0\right\} \cup\{0\}$. Определим функцию $f_{x}: F_{x} \rightarrow \mathbb{R}: \quad f_{x}(u):=\operatorname{sgtr}(\langle X(x), u\rangle) \cdot\langle u, u\rangle^{1 / 2}(\operatorname{sgtr}(0):=0), \quad x \in M$. Конус будушего соответствуюшей линейной кинематики определяется естественным образом: $\forall u \in T_{x} M \quad u \in Q_{x}^{+} \Leftrightarrow u \in F_{x}$ и $f_{x}(u)>0$. Функция $f_{x}$ обладает следующими свойствами:

1) $\forall u, \nu \in Q_{x}^{+}\langle u, \nu\rangle \geqslant f_{x}(u) f_{x}(\nu)$;

2) $\forall u, \nu \in Q_{x}^{+} f_{x}(u+\nu) \geqslant f_{x}(u)+f_{x}(\nu)$;

3) $\forall u \in F_{x}$ и $\forall \alpha \in \mathbb{R} f_{x}(\alpha u)=\alpha f_{x}(u)$.

Иньми словами, пара $\left(T_{x} M, f_{x}\right)$ является псевдометрической линейной кинематикой в смысле определения 5.1 из $[9,10]$. Свойство 2 является неравенством Эйнштейна, которое заменяет неравенство треугольника (аксиома $\mathrm{M}_{2}$ псевдометрической линейной кинематики). Свойство 3 является условием однородности псевдонормы $f_{x}$ (аксиома $\mathrm{M}_{3}$, см. $[9,10])$. Выпуклый конус $Q_{x}^{+}$удовлетворяет аксиоме $\mathrm{M}_{1}($ см. $[9,10])$ :

$$
\forall a \in Q_{x}^{+} \quad \text { и } \forall b \in T_{x} M \quad \exists \alpha \in \mathbb{R}_{+}: a+\alpha b \in Q_{x}^{+} .
$$

Поскольку сигнатура скалярного произведения не зависит от точки, все кинематики $\left(T_{x} M, f_{x}\right)$ линейно изоморфны с сохранением порядка. Обозначим какую-нибудь из этих кинематик через $L$. Функция $f_{x}: F_{x} \rightarrow \mathbb{R}$, строго положительная на $Q_{x}^{+}$и удовлетворяюшая аксиомам $\mathrm{M}_{2}$ и $\mathrm{M}_{3}$, назьвается времениподобной нормой (или псевдонормой) в $T_{x} M$.

Ранее нами была доказана теорема.

Теорема 4. Линейное пространство L с топологией, порожденной псевдонормой $f$, и отношением порядка, порожденным той же псевдонормой, является линейной кинематикой.

Эта теорема была доказана в $[9,10]$ для бесконечномерного пространства $L$. В конечномерном случае она очевидна. Здесь мы докажем, что построенное выше семейство одинаковых конусов может быть задано с помощью кинематического атласа.

Выберем в области $U n$ линейно независимых непрерывных векторных полей, $n:=$ $\operatorname{dim} M$. Приведем матрицу метрического тензора в каждом касательном пространстве 
$T_{x} M$ к каноническому виду $\operatorname{Diag}(1,-1, \ldots,-1,0, \ldots, 0)=V_{x}^{T} g_{x} V_{x}$. Такое приведение $\left(V_{x}\right)$ может быть осуществлено многими способами. В максимальном подпространстве, в котором метрический тензор имеет сигнатуру $(-\cdots-)$, приведение $V_{x}$ определено с точностью до ортогонального преобразования. В максимальном подпространстве, в котором метрический тензор вырожден, это приведение определено с точностью до невырожденного линейного преобразования. Поэтому базис в указанных подпространствах следует выбрать каким-то вполне определенным образом.

Векторы базиса в каждом касательном пространстве представим как линейную комбинацию выбранных ранее векторных полей. Рассмотрим максимальное подпространство, в котором метрический тензор имеет сигнатуру $(-\cdots-)$. Пусть размерность этого подпространства равна $m$. Векторы базиса этого подпространства запишем как столбцы координат. Ранг этой прямоугольной матрицы равен $m$. Выберем из нее невырожденную квадратную матрицу порядка $m$. Окрестность $U$ будем считать настолько малой, чтобы эта подматрица имела максимальный ранг на всем $U$. Выберем базис в рассматриваемом подпространстве так, чтобы в этой подматрице верхний правый угол был нулевым. Размерность группы ортогональных преобразований $O(m)$ как гладкого многообразия равна $m(m-1) / 2$, и мы наложили такое же количество независимых условий на векторы базиса. Чтобы матрица приведения $V_{x}$ была определена однозначно, достаточно фиксировать фазовые множители \pm 1 векторов базиса. Их можно определить из условия $\langle X(x), u\rangle>0$. (Если какой-то вектор базиса $u$ оказался ортогональным $X(x)$, то мы несушественно изменим ориентацию времени $X$, чтобы на $U$ поле $X$ и этот набор векторов не были ортогональны. Изменение ориентации времени будем называть несущественным, если новая ориентация времени лежит в той же самой компоненте связности конуса $F_{x} \backslash\{0\}$ для всех $x \in M$.)

В максимальном подпространстве, в котором метрический тензор вырожден, на векторы базиса нужно наложить $m^{2}$ условий, поскольку размерность группы $G L(m)$ как гладкого многообразия равна $m^{2}$. Потребуем, чтобы координаты векторов базиса этого подпространства образовывали подматрицу (выбираемую так же, как и выше), равную единичной диагональной матрице. Фазовые множители тем самым уже определены.

Касательные пространства в этих новых базисах можно просто отождествить. Отображение $V$, заданное в каждой точке $x \in U$ матрицей $V_{x}$, требуемое. Вообще говоря, эта векторнозначная 1-форма не замкнута. В силу произвольности области $U$ кинематический атлас $P$ построен. Отображения перехода, очевидно, удовлетворяют свойству 3 определения многообразия кинематического типа (и, в частности, условию пункта 2.3.2). Итак, доказана следующая теорема.

ТЕОрема 5. Пусть $M-($ псевдометрическое $)$ многообразие со скалярным произведением $\langle$,$\rangle сигнатуры (+-\ldots-0 \ldots 0)$ и ориентацией времени $X$. Пусть $P-$ построенный выше кинематический атлас. Тогда $(M, P)$ является многообразием кинематического типа $L$, где в качестве $L$ можно взять любое касательное пространство $T_{a} M$ с суженной на него псевдометрикой, причем конус будущего 
этой кинематики выбирается так, чтобы он содержал вектор $X(a)$.

Отметим, что в силу вырожденности метрики на псевдометрическом многообразии, вообше говоря, нет симметричной римановой связности. Аналогом симметричной римановой связности может служить косвязность. Ее символы Кристоффеля определяются соотношением

$$
2 \Gamma_{k \mid i j}=\frac{\partial g_{j k}}{\partial x^{i}}+\frac{\partial g_{k i}}{\partial x^{j}}-\frac{\partial g_{i j}}{\partial x^{k}}
$$

где $\left(g_{i j}\right)_{i, j=1, \ldots, m}$ - матрица метрического тензора. Аналогом ковариантного диффференширования является следуюшая операция:

$$
\frac{\stackrel{*}{D} X}{d t}:=\sum_{j=1}^{m} \frac{d X^{j}}{d t}\left\langle\partial_{j}, \cdot\right\rangle+\sum_{i, j, k=1}^{m} \frac{d \gamma^{i}}{d t} X^{j} \Gamma_{k \mid i j} d x^{k} .
$$

Производная векторного поля есть ковекторное поле. Это определение не зависит от выбора локальных координат.

\section{5. ДОПУСТИМЫЕ КООРДИНАТЫ И ГРУППА ПРЕОБРАЗОВАНИЙ КООР ДИНАТ}

5.1. Допустимые координаты. На многообразиях с отношением локального порядка было бы желательно вводить карты $h: U \rightarrow L$, которые непосредственно сохраняли бы локальный порядок. Однако таких карт, по-видимому, может не быть, т.е. это условие слишком жесткое и может сильно ограничить класс рассматриваемых многообразий и порядков [12]. Решение этого вопроса связано с проблемой пункта 3.2. Можно было бы также потребовать, чтобы дифференциалы карт $d h: T U \rightarrow L$ сохраняли построенное в пункте 2.1 семейство конусов в касательном расслоении данного многообразия. Однако можно показать, что такое условие в действительности эквивалентно требованию, чтобы отображения перехода для самих карт $h_{1} \circ h_{2}^{-1}$ сохраняли порядок в $L$. Поэтому мы будем рассматривать на многообразии $M$ карты вида $h: U \rightarrow L$, не требуя, чтобы локальный порядок в $M$ сохранялся при отображении в $L$. Потребуем меньшего.

Мы видели в пунктах 2.4.5 и 2.3.2, что в линейных кинематиках, слабейшая топология которых содержит антидискретный слой, возникает естественно выделенное подпространство $l_{0}$ - максимальное линейное подпространство, содержашееся в замыкании конуса будушего линейной кинематики. В касательном расслоении $T M$ многообразия кинематического типа $L$ возникает поле линейных подпространств, $l_{x}:=P_{x}^{-1}\left(l_{0}\right)$, $l_{x} \subset T_{x} M \forall x \in M$, т.е. распределение (не зависяшее от выбора элемента $P(U)$ кинематического атласа).

ОПРЕДЕЛЕниЕ. Гладкий атлас $\alpha$ из карт $h: U \rightarrow L$ на многообразии $M$ называется допустимым, если все карты $h \in \alpha$ сохраняют распределение $l: \forall x \in \operatorname{dom} h$ имеем $d_{x} h\left(l_{x}\right)=l_{0}$. 
Если на многообразии $M$ кинематического типа сушествует допустимьй атлас, то координатные функции карт этого атласа можно перенумеровать так, что матрицы дифференциалов всех отображений перехода $h_{1} \circ h_{2}^{-1}$ будут иметь блочный вид, как в пункте 2.3.2. Если указанное распределение голономно, то допустимый атлас сушествует. Достаточно взять в качестве координат со старшими номерами координаты на соответствуюшем интегральном подмногообразии.

Случай, когда размерность конуса будущего кинематики $L$ меньше, чем размерность $L$, рассматривается аналогично.

5.2. Группа преобразований координат. Если на многообразии $M$ есть гладкий атлас $\alpha$ такой, что все его карты имеют дополнительное свойство $\sigma$, то его можно расширить до гладкой структуры $\beta$ всех тех гладко согласованных с $\alpha$ карт, которые обладают тем же свойством $\sigma$. В качестве свойства $\sigma$ мы потребуем, чтобы каждая карта сохраняла определенное выше распределение $\left(l_{x}\right)_{x \in M}$, т.е. $\forall h \in \alpha$ и $\forall x \in \operatorname{dom} h$ имеем $d_{x} h\left(l_{x}\right)=l_{0}$.

ОПРЕДЕЛЕНИЕ. Пусть $M$ - гладкое многообразие с картирующим множеством $L$ и гладкой структурой $\beta$. Преобразованием координат многообразия $M$ мы назовем диффеоморфизм $\varphi: L \rightarrow L$ такой, что для любой карты $h$ из гладкой структуры $\beta$ композиция отображений $\varphi \circ h$ принадлежит той же гладкой структуре.

В выбранной нами гладкой структуре $\beta$ это равносильно требованию, чтобы дифференциал $d \varphi$ сохранял $l_{0}$, т.е. $d \varphi\left(l_{0}\right)=l_{0}$. Следовательно, в определенном выше допустимом атласе матрица Якоби отображения $\varphi$ имеет блочный вид, приведенный в пункте 2.3.2 или 2.3.3, в зависимости от частичного порядка в $L$. Таким образом, мы ограничиваемся теми преобразованиями координат, которые сохраняют некоторую геометрическую структуру на многообразии. Эта геометрическая структура (распределение $\left.\left(l_{x}\right)_{x \in M}\right)$ связана с типом причинности в $M$. Такой подход имеет много общего с предложенной Пименовым полуримановой геометрией [13].

Однако между этими двумя подходами имеется существенное различие. В расслоенных пространствах структурная группа действует на каждом слое. Это так называемая активная точка зрения на преобразования координат. Но действие произвольного элемента структурной группы, вообше говоря, не является изометрией. Поэтому инвариантность по отношению $\kappa$ действию структурной группь приобретает в расслоенных пространствах совсем другой смысл.

Мы придерживаемся так назьваемой пассивной точки зрения на преобразования координат. Определенная выше группа преобразований координат не действует на касательном расслоении многообразия $M$. Она действует на некоторой гладкой структуре многообразия $M$, отображая карту в карту $(h \mapsto \varphi \circ h)$. Такие преобразования не обязаны быть изометриями, и мы можем определять инвариантность по отношению $\kappa$ преобразованиям координат так, как это принято в физике. При таком подходе особое значение приобретает выбор гладкой структуры на многообразии [14-16]. 


\section{Список литературы}

[1] T. I. Kaluza. Sitzungsber. Preuss. Akad. Wiss. 1921. P. 966-972.

[2] V. Fock. Z. Phys. 1926. V. 39. P. 226-232.

[3] O. Klein. Z. Phys. 1926. V. 37. P. 895-906.

[4] Ю. Б. Румер. Исследования по 5-оптике. М.: Гостехиздат, 1956.

[5] A. Einstein, P. Bergmann. Ann. Math. 1938. V. 39. P. 683-701.

[6] H. Busemann. Rozpr. Mat. 1967. № 53. P. 1-50.

[7] Р. И. Пименов. Зап. научн. семин. ЛОМИ. 1968. Т. 6. С. 1-496.

[8] D. Finkelstein. Phys. Today. 1972. V. 25. № 4. P. 51-52; № 8. P. 15.

[9] В. Р. Крыц. Зап. научн. семин. ПОМИ. 1997. Т. 246. С. $152-173$.

[10] V. R. Krym. Causal structures in linear spaces. gr-qc/9704016.

[11] М. А. Улановский. Укр. геометр. сб. 1970. Вып. 7. С. 153-165; Вып. 9. С. 96-110.

[12] E. H. Kronheimer, R. Penrose. Proc. Cambridge Philos. Soc. 1967. V. 63. P. 481-501.

[13] Р.И. Пименов. Труды семинара по векторному и тензорному анализу. 1968. Т. 14. C. $154-173$.

[14] Ю. Д. Бураго, В. А. Залгаллер. Введение в риманову геометрию. Санкт-Петербург: Наука, 1994.

[15] ШІ. Кобаяси, К. Номидзу. Основы дифференциальной геометрии. Т. 1. М.: Наука, 1981.

[16] R. E. Gompf. J. Diff. Geom. 1983. V. 18. № 2. P. 317-328.

Поступила в редакцию 7.VIII.1998 г., после доработки 8.IX.1998 г. 